Mediterránea Ser. Biol. (1987); n. ${ }^{\circ}$ 9. Pág. 87-99

\title{
ESTRUCTURA DE LAS POBLACIONES DE FLEBOTOMOS (DIPT., PSYCHODIDAE) DEL SURESTE DE LA PENÍNSULA IBÉRICA
}

\author{
por \\ E. Martínez Ortega ${ }^{1}$ y E. Conesa Gallego ${ }^{1}$
}

\section{RESUMEN}

Muestreos sobre flebotomos en el sureste de la península Ibérica, han permitido capturar 15.701 ejemplares pertenecientes a ocho especies. De éstas, cuatro son dominantes y las otras cuatro accesorias. Se estudian diferentes aspectos de las comunidades de flebotomos: riqueza específica, distribución altitudinal, fenología, constancia, afinidad interspecífica, convivencia y diversidad específica, lo que permite conocer el funcionamiento y estructura de dichas comunidades.

\section{RESUME}

Une enquete sur Phlébotomes effectuée au sudest de la peninsule Ibérique, nous a permis de récolter 15.701 exemplaires, qui appartient a huit especes, dont quatre prédominent et quatre sont accesoires. Quelques parametres des communautés de Phlébotomes, sont étudies: richesse spécifique, distribution altitudinale, phénologie, fréquence, afinité cénotique, cohabitation et diversité spécifique. Cette étude nous a permis de connaitre le fonctionnement et estructure de ces communautés.

\section{INTRODUCCIÓN}

Los muestreos intensivos de flebotomos en el sureste de la península Ibérica, han permitido la prospección de un elevado número de localidades en las que se ha capturado un número considerable de ejemplares (MARTÍNEZ ORTEGA, 1986) que pertenecen a ocho especies distintas.

La ausencia de trabajos, en la península Ibérica, en los que se realice, de modo general, un estudio integrado de las poblaciones de flebotomos, ha inducido a la realización de este trabajo. En él se exponen resultados de carácter

(1) Departamento Zoología, Facultad de Biología, Universidad de Murcia. 30071 MURCIA, España. 
general que ahondan en el conocimiento de las características del conjunto de las especies y de las relaciones de éstas entre sí. Se analiza el inventario faunístico global y se aplican diversos parámetros y pruebas estadísticas, que permiten obtener una visión de conjunto de la fauna de flebotomos en el área de estudio.

\section{METODOLOGÍA}

Las capturas de flebotomos se han llevado a cabo utilizando, principalmente, la técnica de los papeles adhesivos, trampas clásicas en los muestreos de flebotomos aunque, también, se han usado trampas luminosas de diferentes tipos y la técnica de «cebo humano», que permite la captura de las especies antropófilas. La intensidad de los muestreos y localidades de captura se recogen en un trabajo precedente (MARTÍNEZ ORTEGA, 1986, MARTÍNEZ ORTEGA y CONESA GALLEGO, 1988).

Seguidamente se describen los parámetros y pruebas estadísticas utilizados para el análisis global de los datos, a fin de poder confirmar, de un modo matemático, las observaciones de campo.

La constancia, o frecuencia de aparición de una especie, indica la asiduidad con que se encuentra dicha especie en un muestreo determinado. Esto permite establecer cuatro grupos de especies (TROUILLET, 1981): Muy comunes, con frecuencias superiores al $50 \%$; Comunes, con frecuencias comprendidas entre el $25 \%$ y el $50 \%$; Raras, con frecuencias comprendidas entre el $10 \%$ y el $25 \%$ y Muy raras, cuando presentan frecuencias inferiorés al $10 \%$.

La comparación de la totalidad de las capturas efectuadas en la zona de estudio se ha realizado utilizando el coeficiente de afinidad cenótica de Sorensen, ya empleado anteriormente en el estudio de los flebotomos en la República Popular del Congo (TROUILLET, 1981).

Como convivencia o cohabitación se entiende la probabilidad de que dos especies, elegidas al azar, se encuentren juntas. Para calcular este parámetro hay que conocer el número más probable de muestras en las que dos especies repartidas al azar cohabitan.

Si este valor es mayor que el número de muestras en que se encuentran las dos especies simultáneamente se dice que estas especies son excluyentes; si ambos valores son iguales las dos especies se reparten al azar y finalmente, si el número de muestras comunes es mayor estas especies tienen tendencia a convivir (TROUILLET, 1981).

Para comprobar que esta convivencia es real, se calcula el valor de «Chi cuadrado» que permite estimar la probabilidad de que la convivencia no sea debida al azar del muestreo (DAJOZ, 1975).

La diversidad especifica es un índice que relaciona el número de especies presentes en una comunidad y su abundancia relativa. Para determinarla se utiliza el índice de diversidad de Shannon-Weaver (MARGALEF, 1980), que ya ha sido empleado para el estudio de poblaciones de flebotomos en la República Popular del Congo (TROUILLET, 1981).

El índice de Shannon-Weaver es variable con el número de especies y con el número de individuos de cada una de ellas. Por este motivo, en el caso de los flebotomos, para comparar poblaciones de riqueza diferente, se utiliza la 
equitabilidad, que es la relación entre la diversidad real y la diversidad máxima. La diversidad máxima es la diversidad teórica que se puede esperar cuando todas las especies están representadas por el mismo número de individuos.

\section{RESULTADOS Y DISCUSIÓN}

\section{Inventario faunístico global}

El muestreo, realizado en el área de estudio (sureste de la península Ibérica) empleando los diferentes métodos de captura, ha permitido obtener 15.701 ejemplares, pertenecientes a ocho especies, repartidos como se indica en la tabla 1. De éstos se contabilizan 1.954 ejemplares, pertenecientes a siete especies, que han sido recolectados en la misma zona por el Prof. RIOUX y su equipo. Las técnicas de captura utilizadas para la obtención de estos ejemplares son las mismas que se han usado en el curso de este trabajo.

\section{TABLA I}

\begin{tabular}{lrrrr}
\multicolumn{1}{c}{ ESPECIE } & MACHOS & HEMBRAS & TOTAL & \multicolumn{1}{c}{$\%$} \\
\hline Sergentomyia minuta & 1.716 & 2.325 & 4.042 & $(24,72 \%)$ \\
Phlebotomus papatasi & 1.571 & 311 & 1.882 & $(11,99 \%)$ \\
Phlebotomus alexandri & 293 & 290 & 583 & $(3,71 \%)$ \\
Phlebotomus chabaudi & 22 & 2 & 24 & $(0,15 \%)$ \\
Phlebotomus sergenti & 1.809 & 238 & 2.047 & $(13,04 \%)$ \\
Phlebotomus ariasi & 860 & 38 & 898 & $(5,72 \%)$ \\
Phlebotomus longicuspis & 101 & - & 101 & $(0,64 \%)$ \\
Phlebotomus perniciosus & 5.879 & 245 & 6.124 & $(39,01 \%)$
\end{tabular}

\section{Riqueza específica, abundancia y constancia}

La riqueza de la zona de estudio es, hasta el momento, de ocho especies de las cuales cuatro son dominantes: Phlebotomus perniciosus, Newstead, 1911, Sergentomyia minuta (Rondani, 1843), P. sergenti Parrot, 1917 y P. papatasi (Scopoli, 1786), constituyendo, entre ellas, el $88,76 \%$ del total de las capturas. Las restantes especies: $P$. ariasi Tonnoir, 1921, $P$. alexandri Sinton, $1928, P$. longicuspis Nitzulescu, 1930 y P. chabaudi Crosett, Abonnenc y Rioux, 1970, se pueden considerar accesorias y presentan una menor abundancia (Tabla 1).

Los valores de constancia de cada una de las especies, en la zona de estudio, permite su agrupación del siguiente modo:

-Especies muy comunes: $P$. perniciosus $(88,97 \%), S$. minuta $(76,47 \%)$, $P$. sergenti $(60,29 \%)$.

-Especies comunes: $P$. ariasi $(49,26 \%), P$. papatasi $(47,69 \%)$.

-Especies raras: $P$. longicuspis $(25 \%), P$. alexandri $(10,29 \%)$.

-Especie muy rara: $P$. chabaudi $(4,41 \%)$.

Este agrupamiento está en clara relación con la distribución particular de cada una de las especies. El primer grupo, formado por P. perniciosus, S. mi- 
nuta y $P$. sergenti, lo constituyen especies muy comunes en la península Ibérica y, más concretamente, en la zona sureste de la misma.

Se les puede capturar fácilmente en casi todos los hábitats de esta zona, ya que se trata de especies muy abundantes y ubiquistas, que han sido capturadas desde muy antiguo por diferentes autores (PITTALUGA y DE BUEN; 1917 y 1918; GIL COLLADO, 1927; TORRES CAÑAMARES, 1932; NÁJERA, 1935, etc.).

$P$. ariasi y $P$. papatasi se presentan, en esta zona, en un número más restringido de hábitats y en épocas más concretas, lo que hace que la frecuencia de sus capturas no sea tan elevada como la del grupo anterior.

También se encuentran entre las primeras capturas efectuadas en la península Ibérica (ARIAS ENCOBET, 1911; PITTALUGA y DE BUEN, 1917 y 1918; TONNOIR, 1921, etc.) pero se trata de especies menos ubiquistas. $P$. ariasi se encuentra en las zonas más húmedas y $P$. papatasi en las zonas más áridas.

$P$. longicuspis y $P$. alexandri son especies de distribución restringida y, hasta su captura en la península (RIOUX et al., 1974 y MARTÍNEZ ORTEGA y col., 1982) eran consideradas, en la zona occidental de su distribución, como propias del norte de Africa.

$P$. alexandri ocupa las zonas más áridas de la península y está relegada a una franja próxima a la costa del sureste. $P$. longicuspis es más propia de zonas semiáridas y aparece, con frecuencia, relacionada con $P$. perniciosus, especie a la que sustituye en el Norte de Africa, donde la primera es más abundante que la segunda.

$P$. chabaudi es una especie típicamente norteafricana, que era considerada endémica de dicha región hasta su captura en la península Ibérica (RIOUX et. al., 1974). Tanto en el norte de Africa como en la península; ocupa lugares muy concretos y de condiciones bioclimáticas especiales y su distribución no es muy extensa. Solamente la afinidad bioclimática del área de estudio con el norte de Africa justifica su presencia en ella.

\section{Distribución altitudinal}

Los flebotomos capturados en la zona de estudio colonizan hábitats comprendidos entre $0 \mathrm{~m}$. y $2.168 \mathrm{~m}$. de altitud (Fig. 18).

Después de estandarizar las capturas, refiriéndolas a unidades de superficie, se observa que la mayor parte de individuos se ha recogido entre 0-500 $\mathrm{m}$. Esta zona responde a las condiciones óptimas para la mayoría de las especies. Por encima de los $700 \mathrm{~m}$. de altitud, y hasta $1.100 \mathrm{~m}$., existe un importante número de individuos que corresponde, sobre todo, a las especies de zonas más húmedas. En altitudes superiores a $1.200 \mathrm{~m}$. el número de capturas desciende rápidamente.

La gradación altitudinal de los flebotomos se encuentra en relación directa con las zonas óptimas de desarrollo de las diferentes especie.

Estos datos se ratifican observando la distribución altitudinal del número de especies (Fig. 1A) donde se comprueba que las 8 especies presentes en la zona de estudio se pueden encontrar de 0 a $400 \mathrm{~m}$. de altitud. 


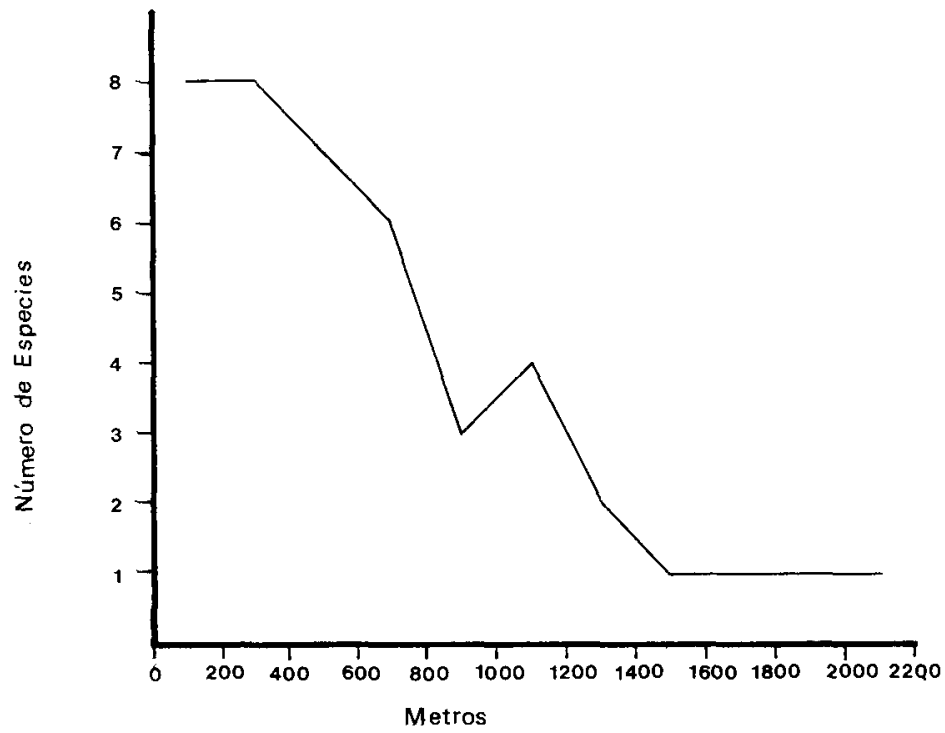

A

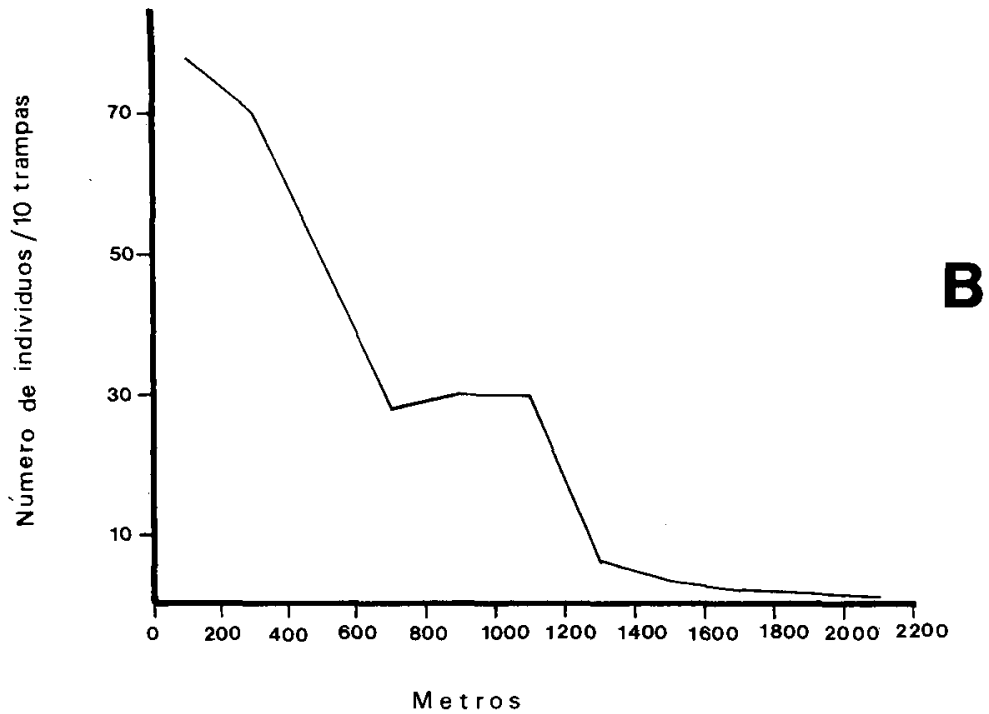

Figura 1.-Distribución altitudinal de los flebotomos. A, distribución del número de especies en función de la altitud. B, distribución del número de individuos capturados en función de la altitud. 
Las altitudes menores $(0-100 \mathrm{~m}$.) están ocupadas, preferentemente, por $P$. papatasi, especie característica de las zonas más secas. Por encima de·los $400 \mathrm{~m}$. ya no aparece la especie $P$. chabaudi y en altitudes superiores a 500 $\mathrm{m}$. no se encuentra $P$. alexandri. En las altitudes intermedias $(100-500 \mathrm{~m}$.) se encuentran las especies de zonas semiáridas $(P$. perniciosus, $P$. longicuspis y S. minuta).

A partir de $800 \mathrm{~m}$. de altitud solamente aparecen 4 especies. $P$. perniciosus, $P$. ariasi, $P$. sergenti y $S$. minuta, aunque esta última no se ha encontrado en las altitudes comprendidas entre 660 y $800 \mathrm{~m}$., debido a que en estas altitudes se localiza un pequeño número de estaciones de muestreo, en las que pueden influir otros factores diferentes a la propia altitud.

$P$. sergenti es la especie más ubiquista y, por esto, se distribuye de un modo casi uniforme entre $0 \mathrm{~m}$. y $1.000 \mathrm{~m}$. de altitud.

Por encima de los $1.200 \mathrm{~m}$. solamente se han realizado capturas de dos especies: $P$. perniciosus y $P$. ariasi. La primera de ellas se ha localizado a una altitud máxima de $1.450 \mathrm{~m}$.

El altitudes superiores a los $1.500 \mathrm{~m}$. se captura, exclusivamente, la especie $P$. ariasi, que es la que alcanza la mayor altitud a la que se han encontrado flebotomos en la península Ibérica $(2.168 \mathrm{~m}$.). $P$. ariasi es la especie típica de las zonas más húmedad y frías lo cual le favorece en su colonización de zonas de alta montaña.

\section{Fenología general}

El conjunto de las especies de flebotomos se encuentra presente durante todo el año en la zona de estudio y su fenología muestra la existencia de dos máximos anuales (Fig. 2), que caracterizan el comportamiento bifásico, típico de las especies de flebotomos presentes en el sureste de la península Ibérica. Esta fenología corresponde a la existencia real de, al menos, dos generaciones anuales distintas.

La primera generación la constituyen los adultos provenientes de las larvas que han pasado el invierno en diapausa (larvas de invierno).

Estos adultos copulan y realizan las puestas que dan lugar a la generación de otoño; ésta la constituyen adultos que, en estado larvario, se han desarrollado durante el verano (larvas de verano). Las puestas de estos adultos dan lugar a las larvas que entran en diapausa, repitiéndose el ciclo.

En las zonas en que existen variaciones climáticas estacionales, como la que nos ocupa, el porcentaje de machos es indicativo del envejecimiento de las comunidades, ya que eclosionan antes que las hembras y mueren, también, antes que ellas (CROSET, 1969 y CROSET et al., 1970).

Por tanto un aumento en el porcentaje de machos, en un momento dado, indica que la población se encuentra en una generación joven, en la que acaban de comenzar las eclosiones de adultos, mientras que un aumento de la proporción de hembras es característica de un envejecimiento de la población de adultos en esa generación.

Para observar cómo varía la relación entre sexos a lo largo del año se ha calculado el porcentaje de machos en cada época de muestreo (Fig. 2).

Se puede observar un aumento en el porcentaje de machos que precede 


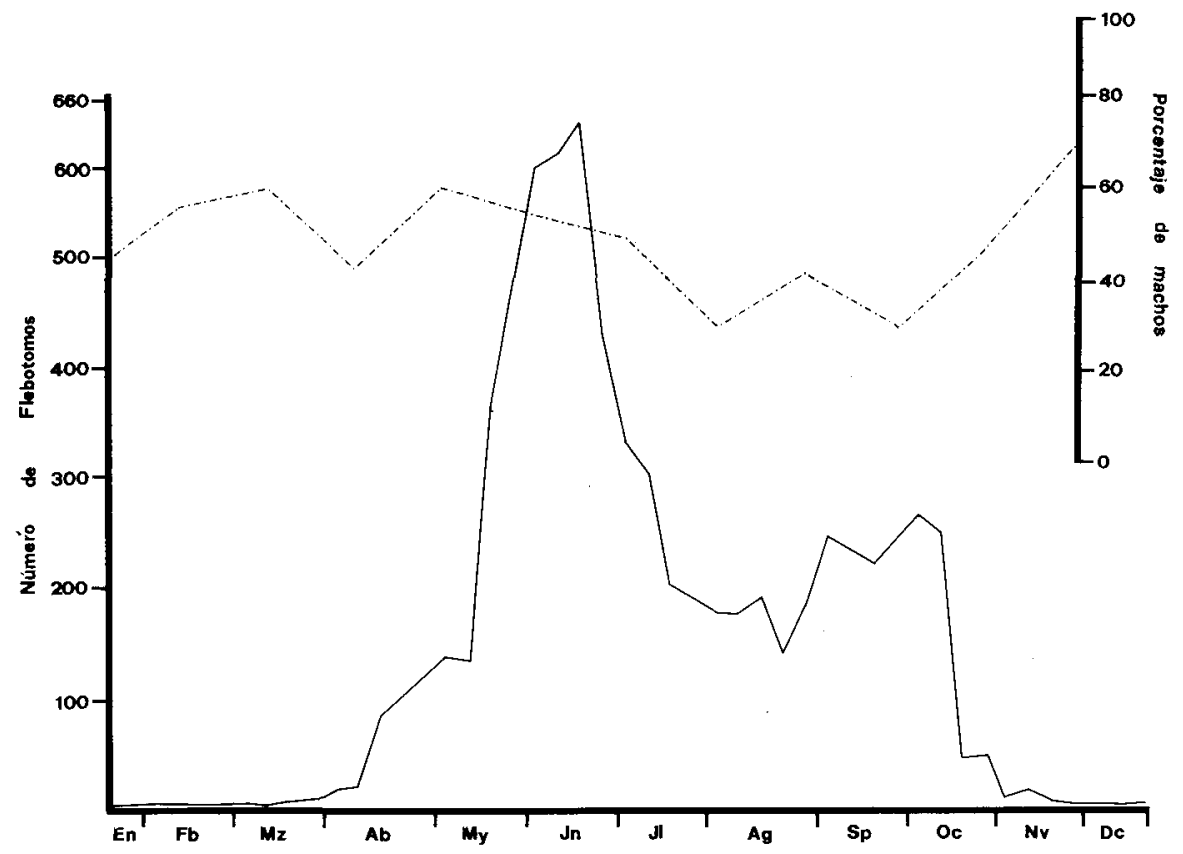

Figura 2.-Fenología general de los flebotomos. Con la línea continua se indica la fluctuación del total de individuos y con la de puntos la fluctuación del porcentaje de machos. El número de flebotomos se refiere al metro cuadrado de superficie.

a los máximos de población y una disminución de dicho porcentaje coincidiendo con los valores mínimos de población.

A partir de octubre comienza un nuevo aumento en el porcentaja de machos que coincide con los meses más fríos. Podría deberse al inicio de la eclosión de adultos de $P$. ariasi, que es la única especie que se mantiene durante todo el año.

Aunque las condiciones termohigrométricas del invierno no permiten la existencia de un gran número de adultos, éstos continúan eclosionando paulatinamente durante este período. Este hecho implica la presencia de un pequeño número de larvas que no entran en diapausa y mantienen un desarrollo continuado hasta llegar al estado adulto.

\section{Afinidad interespecífica. Flebotomocenosis}

Se entiende por flebotomocenosis (TROUILLET, 1981) o población de flebotomos (RIOUX y col., 1984) una agrupación característica de especies pertenecientes a la subfamilia Phlebotominae.

Esta taxocenosis, corresponde a un espacio ecológico particular, que RIOUX y col., 1984 denominan el «espacio leishmánico», el cual está definido no sólo en base a criterios nosogeográficos, sino también en base a determinantes extrínsecos relacionados con los organismos del ciclo epidemiológi- 


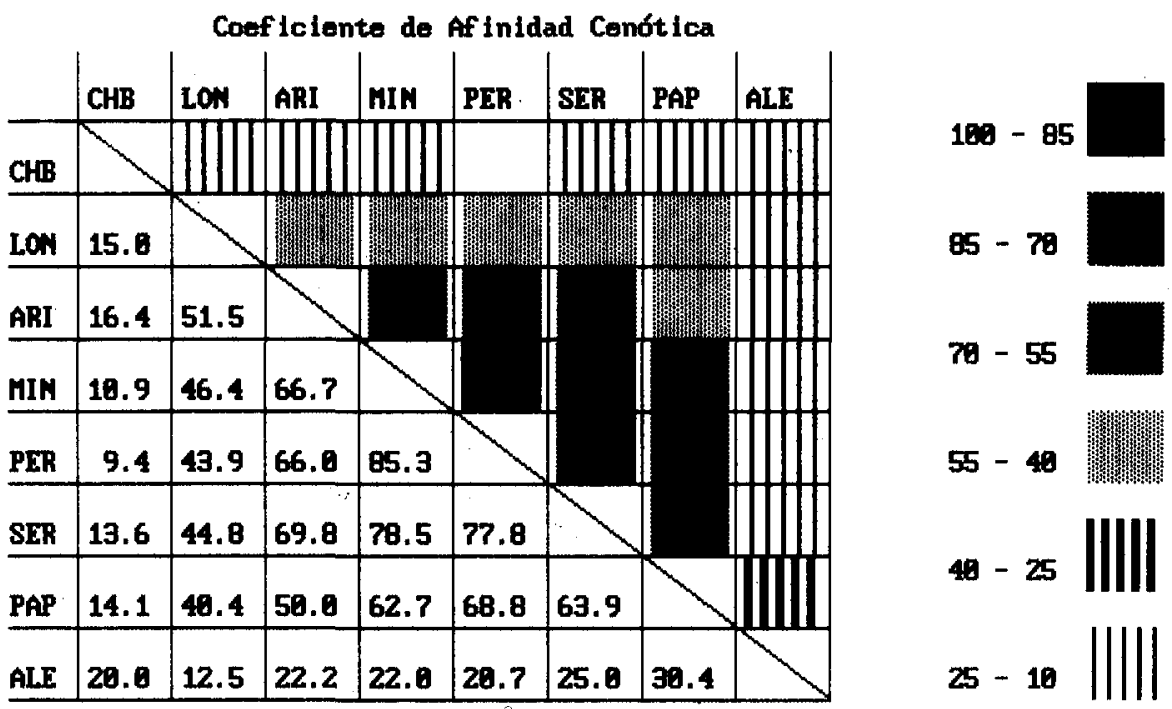

Figura 3.-Representación numérica y gráfica de los valores del coeficiente de afinidad cenótica. Los valores se expresan en porcentaje de afinidad.

co (bioclima, suelo, vegetación, mesoetología de vectores y reservorios, etc.). El cálculo del coeficiente de afinidad para las ocho especies que se encuentran presentes en la zona de estudio, ha permitido identificar una flebotomocenosis (Fig. 3), lo cual ratifica la experiencia de campo.

Existe un primer grupo de especies muy afines, características de la flebotomocenosis y que constituyen el núcleo de la asociación dominante: $S$. minuta, $P$. perniciosus y $P$. sergenti. Estas especies son propias de zonas semiáridas y subhúmedas, pero son muy ubiquistas y de amplia distribución, de modo que se encuentran tanto en zonas áridas como en zonas húmedas, lo cual hace que, frecuentemente, se capturen en los mismos muestreos. Esta es la razón por la que presentan la máxima relación de afinidad.

Este grupo se amplía con dos especies más $P$. ariasi y $P$. papatasi, relacionadas con las anteriores. Se trata de especies más estenoicas, de modo que $P$. ariasi aparece con mayor frecuencia en las zonas más húmedas y $P$. papatasi en las zonas más áridas. La afinidad entre estas dos especies es relativamente baja, debido a que pertenecen a zonas bioclimáticas opuestas, lo cual implica que la posibilidad de que se encuentren en un mismo muestreo es escasa.

$P$. longicuspis se encuentra asociado generalmente a todas las especies anteriores. Se trata de una especie propia de zonas semiáridas y más cercana a $P$. ariasi, con quien presenta la afinidad más alta, mientras que, con $P$. papatasi, su afinidad es la menor de este grupo de especies.

$P$. chabaudi y $P$. alexandri ostentan los valores más bajos de afinidad con el resto de las especies y ellas mismas entre sí. 
La especie $P$. chabaudi se presenta con mayor abundancia en zonas semiáridas, pero su escaso número hace que los valores de afinidad con las restantes especies sean muy bajos.

$P$. alexandri presenta una afinidad algo más elevada con $P$. papatasi que con las demás especies, lo cual caracteriza el grupo de zonas más áridas que, por otra parte, son los lugares óptimos de desarrollo de estas dos especies. Por esto, es más frecuente la relación entre $P$. alexandri y $P$. papatasi que entre Ia primera de ellas y el resto de las especies.

En el área de estudio la gradación entre zonas húmedad y áridas no es tan marcada como para llegar a una separación efectiva de las dos comunidades en dos flebotomocenosis diferentes, pero los datos anteriormente expuestos determinan un principio de separación entre las comunidades de zonas más áridas (representadas por $P$. papatasi) y aquéllas de zonas más húmedad (representadas por $P$. ariasi). De este modo, se perfila la individualización de las comunidades de las dos zonas.

\section{Convivencia entre especies}

El análisis de la convivencia o cohabitación, (Fig. 4), muestra la existencia de una especie, $P$. sergenti, que convive, en mayor o menor proporción, con todas las demás debido a su carácter ubiquista, que hace que se presente con elevada frecuencia en diversos tipos de hábitats.

Luego aparece un grupo integrado por $P$. papatasi y $P$. alexandri, que sólo muestra valores de convivencia, estadísticamente fiables, con $P$. perniciosus y $P$. sergenti.

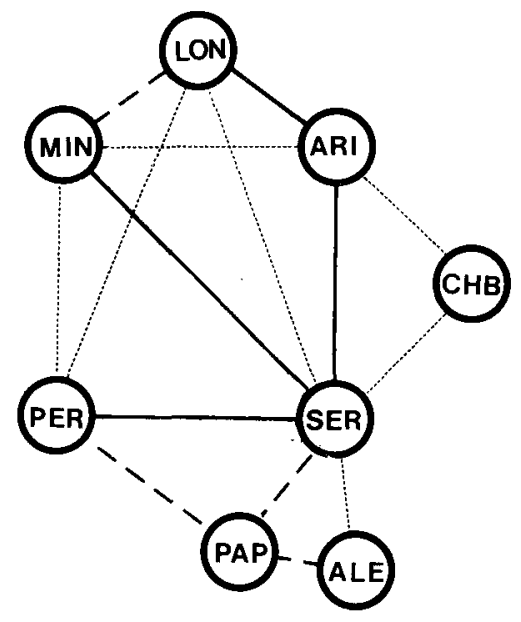

Figura 4.-Representación gráfica de la convivencia entre especies. Las líneas que unen las diferentes especies indican la existencia de convivencia entre ellas. El trazo continuo representa una probabilidad de convivencia de $99,999 \%$; el trazo discontinuo una probabilidad del $99 \%$ y la línea de puntos una probabilidad del $95 \%$ (MIN Sergentomyia minuta, PAP Phlebotomus papatasi, ALE Phlebotomus alexandri, CHB Phlebotomus chabaudi, SER Phlebotomus sergenti, ARI Phlebotomus ariasi, LON Phlebotomus longicuspis, PER Phlebotomus perniciosus). 
La individualización de este grupo, respecto al resto de especies, caracteriza, en base a este nuevo índice, la relación especial entre las dos primeras especies, que son propias de zonas más áridas.

Las demás especies, entre sí, presentan relaciones de cohabitación, más o menos marcadas, que no explican, claramente, ninguna diferenciación en nuevos grupos.

\section{Diversidad específica}

Las comunidades de flebotomos del área estudiada presentan una diversidad de 2.26 y una equitabilidad de 0.75 . Alrededor de estos valores se encuentra, generalmente, la mayoría de las comunidades, ya que el índice utilizado, por su carácter logarítimico, se ajusta a una distribución aproximadamente normal, de modo que el número máximo de comunidades presentan, habitualmente, diversidades entre 1.8 y 2.4 (MARGALEF, 1980).

\section{TABLA II}

REGIÓN O MUESTREO

DIVERSIDAD EQUITAB.

Zaragoza (LUCIENTES CURDI, 1986)

Cataluña española

Francia (CROSET, 1969)

$0,79 \quad 0,34$

Túnez (CROSET, 1969)

$0,98 \quad 0,42$

Granada (MORILLAS MÁRQUEZ, 1982)

Yaka-Yaka (TROUILLET, 1981)

$1,10 \quad 0,47$

Djoumouna (TROUILLET, 1981)

1,22

0,32

1,30

0,46

1,76

0,49

Tata (Sahara) (RIOUX, 1984)

1,85

0,66

SE de la península ibérica

2,19

0,61

Marruecos (BAYLLY-CHOUMARA, 1971)

2,26

2,97

0,75

0,78

Como la diversidad es un valor relativo, su verdadera magnitud se observa al comparar las diversidades de diferentes comunidades.

Hasta el momento, solamente se han utilizado los índices de diversidad en flebotomos, para el análisis de dos flebotomocenosis de la República Popular del Congo (TROUILLET, 1981).

Las diversidades obtenidas son de 1.76, (equitabilidad de 0.49), para las 12 especies presentes en la región de Yaka-Yaka, y de 1.85 (equitabilidad de $0.66)$, para 7 especies presentes en el «bosque galería» de Djoumouna. Comparando con ellas la diversidad obtenida en la zona de estudio ( $\mathrm{Div}=2.26$, $\mathrm{Eq}=0.75$ ), se observa un mayor índice de diversidad en esta última.

Estos datos, aunque a efectos comparativos son válidos, nos proporcionan poca información al tratarse de zonas totalmente diferentes a la nuestra, tanto en el bioclima como en la composición específica y numérica de las flebotomocenosis. Por ello lo ideal es comparar los datos obtenidos en el presente estudio con los de las zonas próximas.

La ausencia de estudios sobre la diversidad en los países circunmediterráneos nos ha llevado a efectuar los cálculos de dichos parámetros en base a da- 
tos que ya obraban en nuestro poder (Cataluña española) y a datos sacados de la bibliografía, cuando la exposición del muestreo realizado hacía posible tal cálculo.

De este modo se han podido obtener los valores de diversidad y equitabilidad de siete nuevas zonas que, con los ya conocidos, se expresan en la tabla 2 . Con estos valores se puede comprobar que la diversidad de la zona estudiada en el presente trabajo es bastante elevada, solamente se presentan con mayor diversidad las comunidades de Marruecos.

Por otra parte, se puede observar que los máximos de diversidad se presentan en la zona sur del Mediterráneo y que estos valores descienden hacia el norte y, de un modo menos acusado, hacia el Ecuador.

Esto refleja que la máxima diversidad, que aparece en la zona sur del Mediterráneo y Norte de Africa, corresponda a un área de interacción entre las comunidades tropicales, etiópicas, (representadas por el género Sergentomyia) y las comunidades mediterráneas, paleárticas, (representadas por el género Phlebotomus). Un ejemplo de esto ocurre en Marruecos donde se entremezclan especies típicamente Paleárticas con otras etiópicas y, en consecuencia, la diversidad es muy elevada.

\section{Fluctuaciones anuales de la diversidad}

Teniendo en cuenta las variaciones anuales de diversidad en un área concreta de la zona de estudio (Fig. 5), se puede ver que la máxima diversidad se alcanza en los meses cálidos (de junio a septiembre). En este período se dan las condiciones óptimas de temperatura y humedad para el desarrollo de los adultos.

En la primavera, la diversidad sufre oscilaciones debido a que esta época coincide con la emergencia de las diferentes especies, por lo que se pasa por un período de estabilización de las comunidades. Los valores más bajos de diversidad de este período coinciden con las temperaturas mínimas más bajas (cf. MARTÍNEZ ORTEGA y CONESA GALLEGO, 1988 para los datos ter-

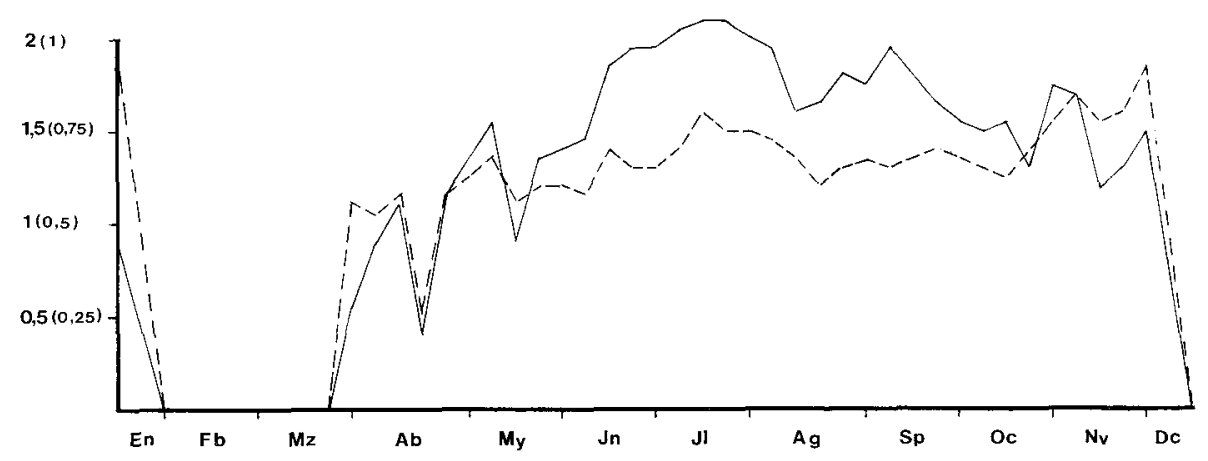

Figura 5.-Representación gráfica de las fluctuaciones anuales de la diversidad (trazo continuo) y equitabilidad (trazo discontinuo). 
mohigrométricos considerados), lo cual indica la gran importancia de la temperatura mínima sobre la diversidad en un período, tan sensible a ella, como el de la eclosión de los adultos.

Durante el otoño la diversidad se mantiene en unos valores relativamente altos, debido a la existencia de varias generaciones lo cual provoca una estabilización de la diversidad y evita que ésta comience a declinar. En esta época la diversidad sufre una serie de oscilaciones, que coinciden con las variaciones de humedad relativa, lo cual indica la importancia de este factor en la viabilidad de los adultos.

Los valores mínimos de diversidad se dan en el invierno (de diciembre a marzo). En estos meses su valor es nulo ya que solamente se encuentra presente una especie: $P$. ariasi que, en la zona de estudio, es propia de las épocas más frías. Este período de diversidad mínima coincide con un descenso de la humedad relativa y de la temperatura, factores importantes para la maduración de larvas y su salida del estado de diapausa.

\section{AGRADECIMIENTO}

Queremos mostrar nuestro agradecimiento al profesor doctor Jean-Antonie Rioux, del Laboratorio de Ecología Médica y Patología Parasitaria de la Facultad de Medicina de Montpellier (Francia), por habernos cedido el material de sus capturas en la Península Ibérica. 


\section{BIBLIOGRAFÍA}

ARIAS ENCOBET, J. (1911), Distribución geográfica de los dípteros en España, Mem. R. Soc. Esp. Hist. Nat., 7: 61-246.

BAILLY-CHOUMARA, H., ABONNENC, E., PASTRE, J. (1971), Contribution a l'étude des Phlébotomes du Maroc (Diptera, Phlebotomidae), Donnes faunistiques et écologiques, $\mathrm{Cah}$. O. R. S. T. O. M., 9: 431-460.

CROSET, H. (1969), Ecologie et Systématique des Phlebotomini (Diptera, Psychodidae) dans deux foyers, français et tunisien, de leishmaniose viscerale. Essai d'interpretation épidémiologique, These Sciences, Montpellier, 516 pp.

CROSET, H., RIOUX, J. A., JUMINER, B., TOUR, S. (1970), Fluctuations anuelles des populations de Phlebotomus perniciosus Newstead, 1911, Phlebotomus perfiliewi Parrot, 1930 et Sergentomyia minuta parroti (Adler et Theodor, 1927) (Diptera, Psychodidae) en Tunisie du Nord, Arch. Inst. Pasteur Tunis, 45: 177-184.

GIL COLLADO, J. (1927), Los insectos hematófagos y transmisores de enfermedades, Publ. Dir. Gen. Sanidad, Madrid: 1-48.

LUCIENTES CURDI, J. (1986), Contribución al conocimiento epidemiológico de la leishmaniosis visceral canina en Zaragoza: Estudio biológico y ecológico de las poblaciones de los flebotomos vectores, Tesis Doctoral, Zaragoza, $449 \mathrm{pp}$.

MARGALEF, R. (1980), Ecología, Ed. Omega, Barcelona, XV+951 pp.

MARTÍNEZ ORTEGA, E. (1986), Los flebotomos (Diptera, Psychodidae) del sureste de la Península Ibérica, Tesis Doctoral, Murcia, 478 pp..

MARTÍNEZ ORTEGA, E., CONESA GALLEGO, E. (1986), los flebotomos (Diptera, Psychodidae) del sureste de la Península Ibérica. Presentación del hábitat y metodología de muestreo, Mediterranea, 9::

MARTÍNEZ ORTEGA, E., WARD, R. D., MARTÍN LUENGO, F., CONESA GALLEGO, E. (1982), Nueva distribución de Phlebotomus (Larroussius) longicuspis Nitzulescu, 1930 (Diptera, Phlebotomidae) en España, Rev. Ibér. Parasitol., 42 (3): 283-288.

MORILLAS MÁRQUEZ, F. (1982), Epidemiología de las leishmaniasis en la provincia de Granada: Estudio biométrico, sistemático y del ciclo anual de los Flebotomos (Diptera, Phlebotomidae), Tesis Doctoral, Granada, $256 \mathrm{pp}$.

NÁJERA ANGULO, L. (1935), Observaciones sobre los «Phlebotomus» recogidos en Sigüenza, Actas Prim. Congr. Nac. Sanidad, Madrid, 1934 (IV): 305-316.

PITTALUGA, G., DE BUEN, S. (1917), Nota sobre los dípteros del género Phlebotomus en España, Bol. Inst. Nac. Hig. Alfonso XIII, 13: 1-9.

PITTALUGA, G., DE BUEN, S. (1918), Especies españolas del género Phlebotomus, Bol. R. Soc. Esp. Hist. Nat., 18: 377-385.

RIOUX, J. A., CROSET, H., LEGER, N. (1974), Présence en Espagne de Phlebotomus alexandri Sinton, 1928 (Diptera, Psychodidae), Ann. Parasitol. Hum. Comp., 49: 126-128.

RIOUX, J. A., CROSET, H., LEGER, N. (1974), Présence en Espagne de Phlebotomus chabaudi Croset, Abonnenc et Rioux, 1970 (Diptera, Psychodidae), Ann. Parasitol. Hum. Comp., 49: 505-507.

RIOUX, J. A., RISPAIL, P., LANOTTE, G., LEPART, J. (1984), Relations Phlébotomesbioclimats en écologie des leishmanioses, Corollaires épidémiologiques. L'exemple du Maroc, Bull. Soc. Bot. Fr., 131: 549-557.

TONNOIR, A. (1921), Une nouvelle espéce européenne du genre Phlebotomus (Phlebotomus ariasi), Ann. Soc. Ent. Belg., 61: 53-56.

TORRES CAÑAMARES, F. (1932), Nota sobre los Phlebotomus y anofelinos de algunos lugares de la provincia de Cáceres, Med. Pais. Cal., 5: 28-32.

TROUILleT, J. (1981), Ecologie des Phlébotomes du Congo (Diptera, Psychodidae), These Sciences, Paris, V+381 pp. 